\title{
WELLENS SYNDROME
}

\author{
Nicole Farmer, MD and Michael Cohen, MD
}

A 76 year-old African-American female with a history of hypertension, type II Diabetes Mellitus, hyperlipidemia, and gatroesphageal reflux presented to emergency room complaining of epigastric pain radiating to left substernum which started the night before presentation. The onset of her sharp pain was at rest, lasted for two hours, and was aggravated by eating and drinking. Her chest pain was followed by gagging and regurgitating of liquid contents. Her chest pain was non-exertional, and not associated with shortness of breath, diaphoresis, nausea, or vomiting. In the Emergency Department, her pain subsided with two sublingual nitroglycerins and 2 milligrams of intravenous morphine. On further questioning the patient stated that the pain had been intermittent for two months. She had seen her outpatient doctor one week prior to admission, who recommended rantidine and an outpatient stress test.

Past cardiac history was significant for a Persantine stress test two years prior that showed normal myocardial perfusion. An echocardiogram done 3 months prior showed asymmetric septal hypertrophy, impaired relaxation of left ventricle, and a low-normal left ventricular systolic function with ejection fraction of 55-60\%.

Physical exam on admission revealed an afebrile, elderly woman with stable vital signs who showed no signs of discomfort. She had normal heart sounds with no murmurs and no reproducible chest pain. Her lung, abdomen and extremity examinations were likewise unremarkable. At the time of admission, her blood work was remarkable for hemoglobin of $12 \mathrm{~g} / \mathrm{dL}$, creatinine of 1.3 $\mathrm{mg} / \mathrm{dL}$, amylase of $97 \mathrm{U} / \mathrm{L}$, lipase of $20 \mathrm{U} / \mathrm{L}$, myoglobin of 143 $\mathrm{ng} / \mathrm{mL}$, and troponin I of $0.50 \mathrm{ng} / \mathrm{mL}$ (reference range 0.05 0.50). A chest x-ray performed in Emergency Department showed no acute disease. The admission EKG is shown below (Figure 1).

Figure 1 shows the EKG on initial presentation when the patient was pain-free. There were new changes when compared to prior EKG. The major finding was biphasic $\mathrm{T}$ waves in V2-V3 with convex morphology of QRS complex. Troponin drawn on arrival was 0.50 (reference range $<0.05$ to 0.50 ). Eight hours after initial presentation, her second troponin returned at 4.09. A subsequent ECG (Figure 2) done, again the patient was pain-free, revealed symmetrical inversion of $\mathrm{T}$ waves in $\mathrm{V} 2$ and $\mathrm{V} 3$.

The patient was taken to cardiac catheterization on the next day and was found to have a $99 \%$ occlusion of left main stem, $99 \%$ occlusion of LAD with active thrombus, and $50 \%$ occlusion of RCA. Patient was emergently taken for CABG, where she underwent grafting of the LAD, obtuse marginal, and PDA.

\section{Discussion}

In retrospect, the patient's history was consistent with unstable angina, although she did manifest atypical symptomatology. Because of her past medical history including GERD and diabetic neuropathy, such comorbidities may have masked true cardiac chest pain. Unfortunately, our patient went on to develop anterior myocardial infarction.

The clinical history and EKG findings are consistent with Wellens syndrome (WS). The syndrome is also referred to as LAD coronary T-wave syndrome. Syndrome criteria include T-wave changes plus a history of anginal chest pain without serum marker abnormalities: no pathological Q waves, no ST segment elevation, and no loss of precordial (V1-V6) R waves. EKG abnormalities of WS appear in one of two ways. The more common variant presents with symmetric and deeply inverted $T$ waves in the precordial leads, usually V2 and V3 (Figure 2). The less common variant presents with biphasic $\mathrm{T}$ waves in the precordial leads, again, usually in V2 and V3 (Figure 1). This case highlights the

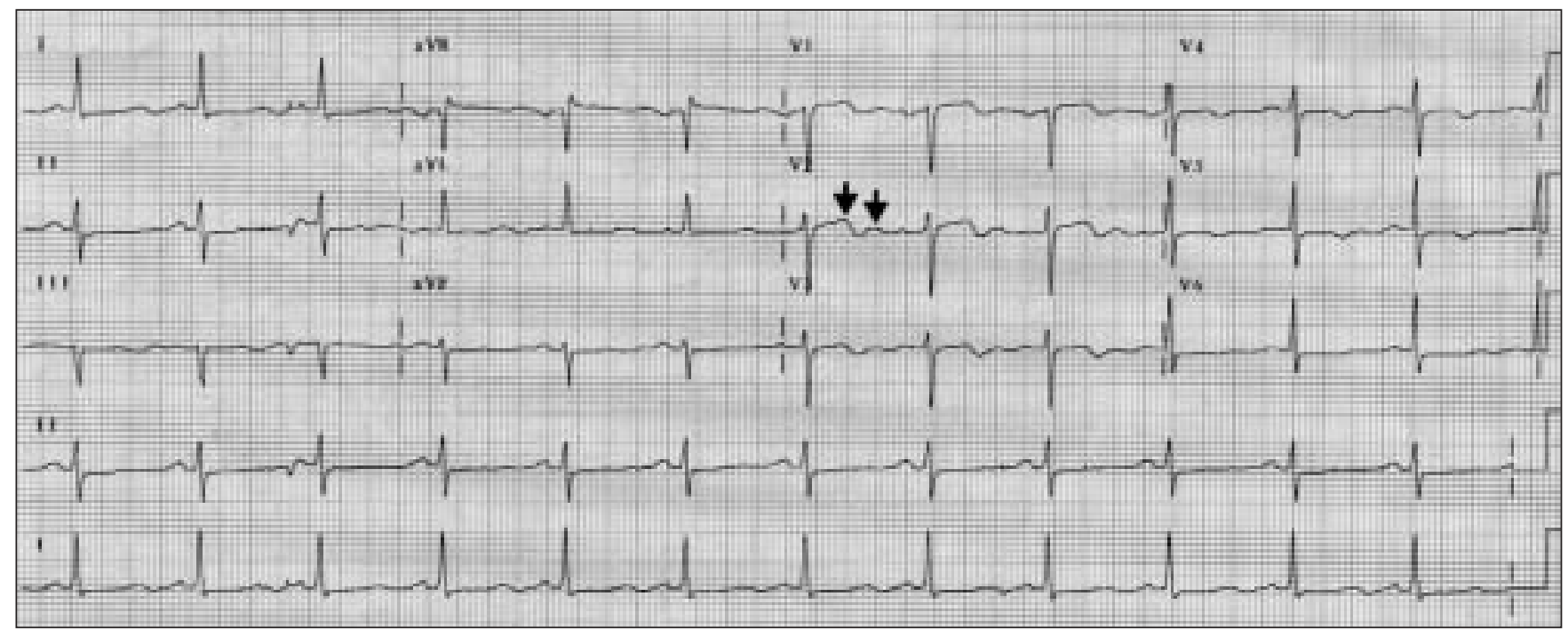

Figure 1. Biphasic T waves in V2 and V3 could easily be mistaken for $u$ waves.

This represents an uncommon variant of Wellens Syndrome. 


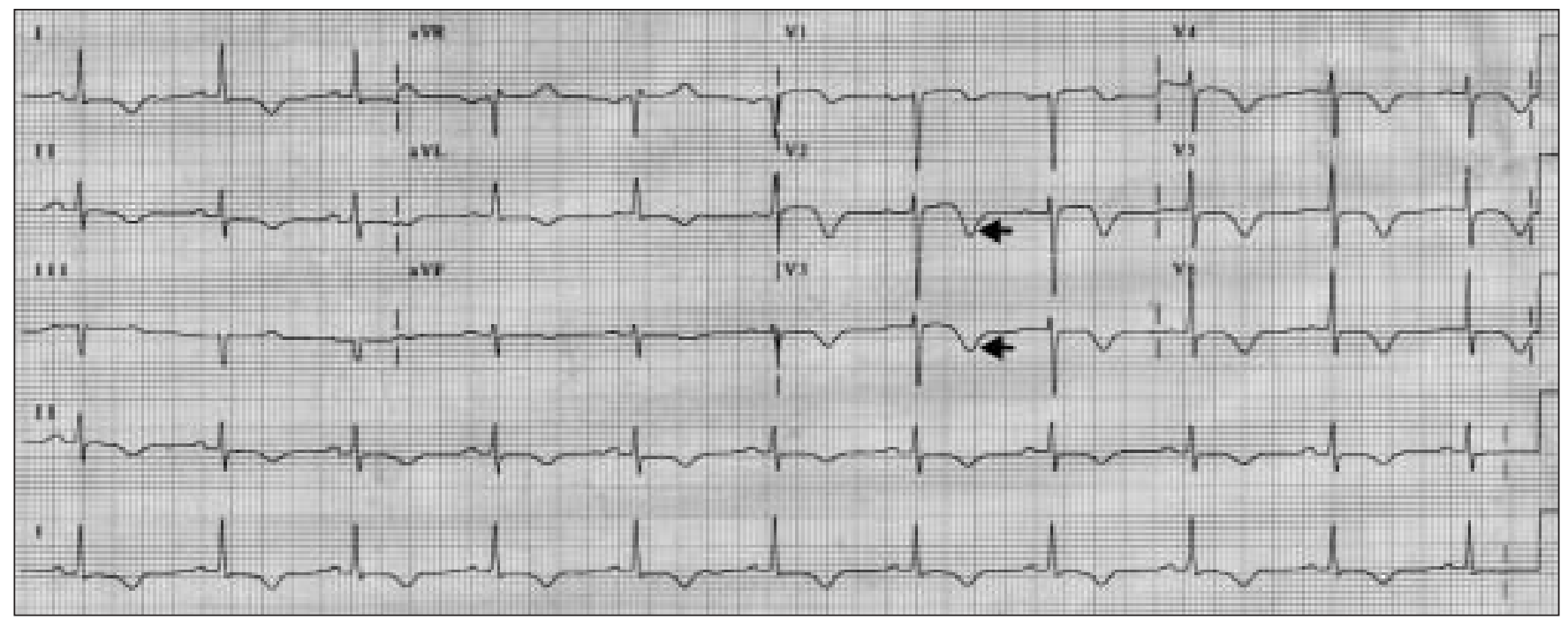

Figure 2. Symmetric inversion of $T$ waves in V2 and V3 is the common variant of Wellens Syndrome.

fact that EKG abnormalities are present in patients even when they do not have the typical chest pain. Moreover, the EKG findings tend to disappear during bouts of angina with normalization of ST segment or T wave abnormalities.

WS was originally described in 1982 by Wellens in his paper with de Zwann and Bar. In this landmark study, $75 \%$ of all patients with WS EKG pattern went on to develop extensive anterior myocardial infarctions within a few days of hospitalization despite management of pain with medical management. In a follow up retrospective study where urgent PCI was performed on all 180 patients meeting WS criteria, 100\% were found to have at least a $50 \%$ obstruction in their LAD artery

It is essential that clinicians recognize that patients admitted to the hospital because of unstable angina who show the characteristic EKG findings described have a high likelihood of having a critical proximal LAD lesion. Such patients are at high risk for the development of an extensive anterior wall MI as was the case for the patient described. In this subgroup of patients, urgent coronary angiography and, when possible, coronary revascularization should be undertaken. Furthermore, exercise stress tests are contraindicated due to presence of extensive lesions and several case reports of death following treadmill stress testing.

\section{References}

1. de Zwaan C, Bar FW, Wellens HJ. "Characteristic electrocardiographic pattern indicating a critical stenosis high in left anterior descending coronary artery in patients admitted because of impending myocardial infarction.” American Heart Journal 1982;103(4 pt 2):730-6.

2 de Zwann C, Bar FW, Janssen JH, Cheriex EC, Dassen WR, Brugada P, et al. "Angiographic and clinical characteristics of patients with unstable angina showing an EKG pattern indicating critical narrowing of the proximal LAD coronary artery." American Heart Journal 1989;117:657-65.

3. Haines DE, Raabe DS, Gundel WD, Wackers FJ. "Anatomic and prognostic significance of new T-wave inversion in unstable angina." American Journal of Cardiology 1983;52:14-8.

4. Tandy TK, Bottomy DP, Lewis JG. "Wellens' syndrome.” Annals of Emergency Medicine 1999;33:347-51. 